\title{
Effet du régime et de la teneur en protéines brutes alimentaires sur le rendement en viande de l'escargot Achatina fulica (Bowdich, 1720)
}

\author{
Nygblé Angèle SIKA PIBA ${ }^{1}$, Mamadou KARAMOKO ${ }^{1 *}$, \\ Coffi Franck Didier ADOU ${ }^{2}$, Atcho OTCHOUMOU ${ }^{1}$ et Kouassi Philippe KOUASSI ${ }^{3}$ \\ 1 Laboratoire de Biologie et Cytologie Animales, Université Nangui Abrogoua, \\ BP 801 Abidjan 02, Côte d'Ivoire. \\ 2 Département des Sciences et Technologie, Ecole Normale Supérieure-Cocody, \\ Abidjan, 08 BP 10 Abidjan 08, Côte d'Ivoire. \\ ${ }^{3}$ Laboratoire de Zoologie-Biologie Animale, Université Félix Houphouët Boigny de Cocody, \\ 01 BP V34 Abidjan 01, Côte d'Ivoire. \\ *Auteur correspondant,E-mail : petyhabib@yahoo.fr
}

\section{RESUME}

Le rendement en viande de l'escargot $A$. fulica soumis à deux régimes végétaux de fourrages verts $\left(\mathrm{R}_{1}\right.$ et $\left.\mathrm{R}_{2}\right)$ et 3 régimes concentrés de farines $\left(\mathrm{R}_{3}, \mathrm{R}_{4}\right.$ et $\left.\mathrm{R}_{5}\right)$ avec différentes teneurs en protéines (10,5\%; $14 \%$ et $17,5 \%$ ), a été évalué après 50 semaines d'élevage en milieu expérimental. Le régime $\mathrm{R}_{1}$ est constitué de lactuca sativa (Asteraceae), de carica papaya (Caricaceae), Brassica oleracea (Brassicaceae), de Cecropia peltata (Moraceae), Laportea aestuans (Urticaceae) et de Phaulopsis falcisepala (Acanthaceae). Le régime $\mathrm{R}_{2}$, en plus des feuilles utilisées pour le régime $\mathrm{R}_{1}$, est additionné de feuilles de Leucena leucocephala (Fabaceae-Mimosoideae), une plante relativement riche en protéines. Les animaux soumis aux régimes constitués de fourrages verts ont présenté des quantités de chair consommables $(52,58$ g pour $R_{1}$ et $57,90 \mathrm{~g}$ pour $R_{2}$ ) plus faibles que celles de ceux soumis aux régimes concentrés de farine [62,88 $\mathrm{g}\left(\mathrm{R}_{3}\right)$; 76,81 $\mathrm{g}\left(\mathrm{R}_{4}\right)$ et 85,45 $\left.\mathrm{g}\left(\mathrm{R}_{5}\right)\right]$. Le régime $\mathrm{R}_{5}$ présentant la plus forte teneur en protéines $(17,5 \%)$, a induit la plus importante quantité de chair consommable $(85,45 \mathrm{~g})$. En revanche, la plus faible quantité de viande est fournie par les escargots soumis au régime végétal $\mathrm{R}_{1}$ sans $L$. leucocephala avec la plus faible teneur en protéines $(2,75 \%)$. Ainsi, la quantité de viande des escargots, s'est accrue avec la teneur en protéines du régime alimentaire.

(c) 2014 International Formulae Group. All rights reserved.

Mots clés: A. fulica, régime alimentaire, protéines, performance de croissance, chair

consommable.

\section{INTRODUCTION}

Les escargots sont beaucoup appréciés pour la quantité de viande qu'ils procurent. La partie du corps généralement consommée est la masse pédieuse (Otchoumou et al., 2010). La coquille et la masse viscérale peuvent être revalorisées en nutrition animale (Otchoumou, 2005). Si la viande de cet animal est tant appréciée et de plus en plus consommée par les populations ivoiriennes, c'est en partie grâce à de son parfum et de sa saveur (Kouassi et al., 2007). Pourtant, cette viande renferme 
d'importantes qualités nutritives. En effet, c'est une importante source de protéine, d'acides aminés (lysine, phénylalanine et leucine), d'énergie et de matières minérales (Fagbuaro et al., 2006; Diomandé et al., 2008; Otchoumou et al., 2010). Par contre, la chair de ces mollusques est très pauvre en lipide. C'est à juste titre qu'elle est conseillée en diététique dans les régimes pauvres en lipide (Engmann et al., 2013). La portion de chair traditionnellement consommée par l'homme en Afrique Occidentale représente environ un tiers du poids vif de l'escargot géant contre près de la moitié du poids vif pour la coquille et les viscères non consommés (Sami et al., 2004; Otchoumou, 2005). On peut ainsi, récupérer pour un kilogramme d'escargot géant destiné à la consommation humaine, près d'un demi-kilogramme de tissus de grande valeur pour l'alimentation animale (calcium et protéine).

Une étude de l'effet d'un déséquilibre en acides aminés d'aliments concentrés sur les préférences alimentaires de Cepaea nemoralis et Achatina fulica a montré que les deux espèces préféraient l'aliment concentré complet par rapport aux aliments déséquilibrés en nutriments (Ademolu et al., 2004). Par contre, aucune étude approfondie n'a encore été menée sur le taux minimal de protéines à incorporer dans le régime alimentaire concentré des escargots A. fulica pour garantir le succès de leur élevage. C'est pourquoi cette étude se propose d'évaluer l'effet du régime et du taux de protéines alimentaires sur le rendement en viande de l'escargot Achatina fulica.

\section{MATERIEL ET METHODES}

\section{Cadre expérimental}

Cette étude a été réalisée au centre d'achatiniculture de l'Université Nangui Abrogoua (Abidjan, Côte d'Ivoire). Ce centre comporte un bâtiment où l'élevage se fait sous abris et une zone d'expérimentation en plein air. La température et l'humidité relative mensuelles moyennes dans le bâtiment d'élevage ont été respectivement de 26,7 \pm $1,4{ }^{\circ} \mathrm{C}$ et de $82,6 \pm 1,4 \%$. La photopériode a été 12 heures de lumière et 12 heures d'obscurité.

\section{Animaux}

Les animaux utilisés dans ce travail sont des Mollusques, Gastéropodes, Pulmonés. Ils appartiennent à l'ordre des Stylommatophores, à la super famille des Achatinaceae, à la famille des Achatinidae, au genre Achatina et à l'espèce Achatina fulica (Bowdich, 1720).

\section{Enceintes d'élevage}

Les escargots ont été élevés dans des bacs en matière plastique de longueur 0,66 $\mathrm{m}$, de largeur $0,6 \mathrm{~cm}$ et de hauteur $0,2 \mathrm{~m}$ soit une surface de base d'environ $0,4 \mathrm{~m}^{2}$ et un volume de $0,08 \mathrm{~m}^{3}$. Ces enceintes sont dotées de couvercle de type moustiquaire constituant un dispositif antifuite. Leur fond est recouvert de terreau à une hauteur de $4 \mathrm{~cm}$ d'épaisseur.

\section{Méthodes}

Deux cent vingt-cinq (225) escargots Achatina fulica d'environ trois jours d'âge ont été repartis de façon aléatoire suivant cinq (5) régimes alimentaires dont deux constitués de fourrages verts $\left(R_{1}\right.$ et $\left.R_{2}\right)$ et trois concentrés de farine $\left(R_{3}, R_{4}\right.$ et $\left.R_{5}\right)$. Ces animaux ont été repartis en raison de 3 répétitions par régime. Le régime végétal de fourrages verts $R_{1}$ a été constitué de feuilles de lactuca sativa (Asteraceae), de carica papaya (Caricacea), Brassica oleracea (Brassicaceae), de Cecropia peltata (Moracae), Laportea aestuans (Urticaceae) et de Phaulopsis falcisepala (Acanthaceae) (Tableau 1). Le régime $\mathrm{R}_{2}$, en plus des feuilles utilisées pour le régime $\mathrm{R}_{1}$, a été additionné de feuilles de Leucena 
leucocephala (Fabaceae-Mimosoideae), une plante relativement riche en protéines.

Quant aux régimes concentrés de farine, ils ont été formulés en variant leur teneur en farine de soja, source de protéine (Tableau 2). Pour éviter la variation simultanée du taux des autres nutriments suite à la diminution du taux de la source protéique dans les régimes $R_{3}$ et $R_{4}$, de l'Agar-agar en poudre a été incorporé à ceux-ci. En effet, aux régimes $R_{3}$ et $R_{4}$ ont été ajoutés respectivement, $15 \%$ et $10 \%$ de ce composé afin d'ajuster leur composition centésimale à $100 \%$. Ainsi, les proportions de farine de soja graine dans les régimes $\mathrm{R}_{3}, \mathrm{R}_{4}$ et $\mathrm{R}_{5}$ sont respectivement de 5\%,10\%, 20\%. Ce qui a permis d'obtenir des régimes avec des teneurs en protéine variables $(10,5 \%$; $14 \%$ et $17,5 \%$ ).

Les aliments sont pesés avant d'être servis aux animaux tous les deux jours. Au terme des deux jours, les refus alimentaires sont pesés et les mangeoires proprement lavées avant d'être réutilisées. Pour chaque aliment servi, un témoin de $100 \mathrm{~g}$ est placé dans les mêmes conditions expérimentales dans des bacs ne contenant pas d'animaux. La pesée de ces aliments témoins au même moment que les refus alimentaires, permet de faire les corrections de poids dues à la dessiccation pour le régime végétal de fourrages verts et à l'hydratation pour les régimes concentrés de farine. Les ingestions alimentaires, les taux moyen journalier de consommation et les rendements écologiques de croissance en fonction des régimes et des taux de protéines brutes alimentaires ont été calculés selon les formules suivantes:

$$
\mathbf{I}_{\mathbf{A}}(\mathrm{g} / \mathrm{j} / \text { escargot })=\frac{\left(\mathrm{I}_{2}-\mathrm{I}_{1}\right)-|\mathrm{Paf}-\mathrm{Pai}|}{\mathrm{Tf}-\mathrm{Ti} \mathrm{Ti}}
$$

Avec:

$\mathbf{I}_{\mathbf{A}}=$ Ingestion alimentaire

$\mathrm{I}_{1}=$ quantité d'aliment servi ;

$\mathrm{I}_{2}=$ quantité du refus alimentaire ;
Pai $=$ poids aliment témoin initial ;

Paf = poids aliment témoin final ;

$\mathrm{Ti}=$ temps initial ;

$\mathrm{Tf}=$ temps final

Après 50 semaines d'élevage, trente escargots ont été prélevés au hasard par régime alimentaire pour l'évaluation de leur rendement en viande. Après 24 heures de jeûne, les escargots sélectionnés ont été marqués, mesurés et pesés avant d'être euthanasiés par ébouillantage (immersion dans l'eau bouillante pendant $10 \mathrm{~min}$ ). Après refroidissement, les tissus mous ont été retirés des coquilles, puis égouttés sur du grillage. Les coquilles vides ont été également égouttées. Un ensemble d'organes (pied, tête, bord distal du manteau portant le dernier centimètre du rectum et l'anus, le pneumostome, le bourrelet palléal, la partie distale du tractus génital y compris le manchon pénien, la partie antérieure du tractus digestif jusqu'à la première moitié du jabot) correspondant à la portion généralement consommée en Côte d'Ivoire, a été séparé du reste des organes viscéraux (glandes digestives, gonades, glandes à albumine, conduit génital, cœur et hépatopancréas). Pour chaque escargot, le poids de l'ensemble des chairs, celui de la masse pédieuse, de la masse viscérale et le poids de la coquille vide, ont été déterminés à l'aide d'une balance de marque Sartorus. Ces pesées ont permis de calculer les proportions des différentes parties du corps des escargots (masse pédieuse, masse viscérale, coquille vide et tissus mous) selon les formules suivantes :

- Proportion de coquille $(\%)=\mathrm{P}_{\mathrm{Cq}} \times 100 /$ PV

- Proportion de masse viscérale $(\%)=100$ $\mathrm{P}_{\mathrm{Vc}} / \mathrm{PV}$

- Proportion de tissus mous $(\%)=100 \mathrm{P}_{\mathrm{Tm}} /$ PV

- Proportion de masse pédieuse $(\%)=$ $100 \mathrm{P}_{\mathrm{mp}} / \mathrm{PV}$

où

$\mathrm{P}_{\mathrm{Cq}}$ : Poids coquille vide en $\mathrm{g}$ 
PV : Poids vif de l'escargot en g

$\mathrm{P}_{\mathrm{Vc}}$ : Poids de la masse viscérale en $\mathrm{g}$

$\mathrm{P}_{\mathrm{Tm}}$ : Poids des tissus mous en $\mathrm{g}$

$\mathrm{P}_{\mathrm{mp}}$ : Poids de la masse pédieuse en $\mathrm{g}$

\section{Analyses statistiques}

Les différents traitements statistiques ont été effectués à l'aide du logiciel STATISTICA version 7.1. Les poids vifs et les quantités des différentes parties du corps des escargots en fonction du régime et du taux de protéine alimentaire ont été comparés grâce au test LSD à un seuil de confiance de 5\%. Les proportions corporelles de ces escargots ont été comparées grâce au test HSD de TUKEY à un seuil de confiance de 5\%. Quant aux compositions biochimiques des régimes alimentaires, elles ont été comparées en utilisant le test de Kruskal-Wallis à un seuil de confiance de $5 \%$.

\section{RESULTATS}

\section{Caractéristique chimique des régimes alimentaires}

Les valeurs nutritionnelles des régimes végétaux de fourrages verts et concentrés sont présentées par le Tableau 3.

Les régimes végétaux ont un taux d'humidité $\left(89,27 \%\right.$ pour $\mathrm{R}_{1}$ et $76,5 \%$ pour $\mathrm{R}_{2}$ ) supérieur aux régimes concentrés de farine ( $13 \%$ pour $\mathrm{R}_{3} ; 6,31 \%$ pour $\mathrm{R}_{4}$ et $6,64 \%$ pour $R_{5}$ ). Cependant, les régimes concentrés sont plus riches en nutriments et en énergies métabolisables que les régimes végétaux de fourrages verts. Les taux de protéines sont respectivement de 10,5\%, $14 \%$ et $17,5 \%$ dans les régimes concentrés contenant $5 \%\left(\mathrm{R}_{3}\right) ; 10 \%\left(\mathrm{R}_{4}\right)$ et $20 \%\left(\mathrm{R}_{5}\right)$ de farine de soja. Des deux régimes végétaux, celui contenant les feuilles de leucena leucocephala $\left(\mathrm{R}_{2}\right)$ est le plus riches en protéine avec un taux de $4,94 \%$ contre 2,75\% pour le régime sans Leucena. Dans les régimes concentrés, le taux de matière minérale variant entre 28 et $40 \%$ est supérieur à celui de protéine brute compris entre $10,5 \%$ et $17,5 \%$. Par contre, le taux de protéine brute dans les régimes végétaux, est supérieur à celui de la matière minérale.

\section{Ingestion alimentaire}

L'ingestion alimentaire, le taux moyen journalier de consommation et le rendement écologique de consommation en fonction du régime et du taux de protéine alimentaire de A. fulica sont regroupés dans le Tableau 4. La quantité d'aliment concentré de farine journellement consommée par escargot varie entre 0,78 et $1 \mathrm{~g} / \mathrm{j}$. Quant à celles des régimes constitués de fourrages verts $R_{1}$ et $R_{2}$, elles sont respectivement de 1,24 et $1,89 \mathrm{~g} / \mathrm{j} /$ escargot. Aucune différence statistique n'est révélée entre les ingestions alimentaires des animaux soumis aux régimes concentrés de farine. En revanche, elles sont statistiquement inférieures à celles des animaux soumis aux régimes constitués de fourrages verts $\left(R_{1}\right.$ et $\left.R_{2}\right)$. Aussi, les taux moyens de consommation journaliers des régimes végétaux de fourrages vets $(6,45 \%$ pour $\mathrm{R}_{1}$ et $5,79 \%$ pour $\mathrm{R}_{2}$ ) sont significativement supérieurs à ceux des régimes concentrés de farine $(2,26 \%$ pour $\mathrm{R}_{3} ; 2,03 \%$ pour $\mathrm{R}_{4}$ et $1,79 \%$ pour $\mathrm{R}_{5}$ ) statistiquement identiques. Par contre, le rendement écologique de consommation des régimes concentrés est nettement supérieur à celui des régimes végétaux de fourrages verts. En effet, le rendement écologique de croissance des régimes concentrés de farine varie entre 48,33 et $61 \%$ alors que ceux des régimes végétaux de fourrages verts $\mathrm{R}_{1}$ et $\mathrm{R}_{2}$ sont respectivement de $16,37 \%$ et de $22,5 \%$. L'analyse des résultats indique un accroissement du rendement écologique de croissance avec la teneur en protéines du régime alimentaire.

\section{Rendement en viande}

L'effet de la variation du régime et du taux de protéine alimentaire sur les quantités de tissu mou et de coquille produites par A. fulica est résumé dans le 
Tableau 5. Les animaux aux poids vifs compris entre 119,47 et 198,6 g présentent des quantités de coquille comprises entre 24,98 et $49,03 \mathrm{~g}$ puis des quantités de tissu mou comprises entre 83,46 et $132,91 \mathrm{~g}$. La plus importante quantité de viande $(85,45$ g) est obtenue avec le régime concentré $R_{5}$ (17,5\% de protéine) et la plus faible $(52,58$ g) avec le régime constitué de fourrages verts sans $L$. leucocephala $\mathrm{R}_{1}(2,5 \%$ de protéine). L'analyse statistique indique une augmentation significative de la quantité de coquille; de tissu mou total et de chair consommable avec le taux de protéine brute alimentaire. Les escargots produits au cours de cette expérience présentent des proportions coquillières comprises entre 20,93 et 24,7\% (Tableau 6). Quant à leurs proportions de tissus mous, elles ont pour valeurs extrêmes $66,9 \%\left(\mathrm{R}_{5}\right)$ et $70,03 \%$ $\left(\mathrm{R}_{2}\right)$. Les animaux soumis aux régimes constitués de fourrages verts, présentent des proportions de coquille $(20,93 \%)$ plus faibles que ceux soumis aux régimes concentrés de farine $\left(23,23 \%\right.$ pour $\mathrm{R}_{3}$; $23,57 \%$ pour $\mathrm{R}_{4}$ et $24,70 \%$ pour $\mathrm{R}_{5}$ ). Par contre, ceux-ci ont des proportions de tissus $\left(69,83 \%\right.$ pour $R_{1} ; 70,03 \%$ pour $\left.R_{2}\right)$ plus importantes que ceux des régimes concentrés $\left(68,5 \%\right.$ pour $\mathrm{R}_{3} ; 68,03 \%$ pour $\mathrm{R}_{4}$ et $66,9 \%$ pour $\mathrm{R}_{5}$ ). Les rendements en viande et en viscère enregistrés au cours de cette étude varient respectivement entre 43 et $43,97 \%$ et entre 23,9 et $26,77 \%$. L'analyse statistique n'indique aucune différence significative entre les différents régimes en ce qui concerne ces deux paramètres.

\section{Composition biochimique des masses pédieuse et viscérale}

La teneur en protéines des masses pédieuses des escargots soumis aux régimes concentrés $\mathrm{R}_{3} \quad\left(10,5 \%\right.$ de protéine); $\mathrm{R}_{4}$ (14\% de protéine) et $R_{5} \quad(17,5 \%$ de protéine) varient de 63,07 à $65,74 \%$
(Tableau 7). Celles de leurs masses viscérales varient de 56,49 à 59,85\%. Quant aux animaux soumis aux régimes constitués de fourrages verts, $R_{1}(2,75 \%)$ et $R_{2}$ $(4,94 \%)$, leurs masses pédieuses présentent des teneurs en protéine respectives de $58,96 \%$ et $60,83 \%$. La masse viscérale de ces animaux a une teneur en protéine de $52,88 \%$ pour le régime $R_{1}$ et de $56,12 \%$ pour $R_{2}$. Les masses pédieuse et viscérale des escargots sauvages offrent des taux de protéine brute respectifs de $67,9 \%$ et $61,49 \%$. Pour tous ces animaux, la teneur en protéine de la masse pédieuse est légèrement supérieure à celle de la masse viscérale. Aussi, la chair des animaux sauvages, présente une teneur en protéine légèrement supérieur à celles de ceux d'élevage. En milieu d'élevage, ceux soumis aux régimes concentrés sont légèrement plus riches en protéine que ceux soumis aux fourrages verts. La teneur en matières minérales de la masse pédieuse des animaux de la nature est de $7,56 \%$ et celle de leur masse viscérale est de $8,86 \%$. La valeur énergétique de leurs masses pédieuses est de $4967 \mathrm{cal} / 100 \mathrm{~g}$ contre 4898 cal/100 g pour la masse viscérale. La teneur en matière minérale de la masse pédieuse des animaux soumis au régime végétal $R_{1}$ est de $4,02 \%$ et de $3,75 \%$ pour ceux soumis au régime végétal $R_{2}$. La valeur énergétique de la masse pédieuse est de $5012 \mathrm{cal} / 100 \mathrm{~g}$ avec le régime $\mathrm{R}_{1}$ et de 4992 cal/100 g avec le régime $R_{2}$. Les escargots soumis aux régimes concentrés présentent quant à eux une teneur en matières minérales au niveau de la masse pédieuse variant de 14,98 à $15,19 \%$ et au niveau de la masse viscérale entre $16,77 \%$ et $17,23 \%$. La valeur énergétique de la masse pédieuse de ces animaux varie de $5101 \mathrm{cal} / 100 \mathrm{~g}$ à $5406 \mathrm{cal} / 100$ g. En revanche, celle de leurs masses viscérales est comprise entre 4865 et 5002. 
Tableau 1: Composants des régimes concentrés.

\begin{tabular}{lccc}
\hline Constituants (g) & \multicolumn{3}{c}{ Régimes } \\
\cline { 2 - 4 } & $\mathbf{R}_{\mathbf{3}}$ & $\mathbf{R}_{\mathbf{4}}$ & $\mathbf{R}_{\mathbf{5}}$ \\
\hline Maïs & 15,3 & 15,3 & 15,3 \\
Tourteaux de coton & 16 & 16 & 16 \\
Soja graines & 5 & 10 & 20 \\
Blé tendre remoulage bis & 15 & 15 & 15 \\
Phosphate bi calcique & 4 & 4 & 4 \\
Complexe vitaminé & 0,5 & 0,5 & 0,5 \\
Carbonate de calcium & 28,7 & 28,7 & 28,7 \\
Sel & 0,4 & 0,4 & 0,4 \\
Oligo-éléments & 0,1 & 0,1 & 0,1 \\
Agar-agar & 15 & 10 & 0 \\
Total (g) & 100 & 100 & 100 \\
\hline
\end{tabular}

Tableau 2 : Composants des régimes végétaux.

\begin{tabular}{lcc}
\hline & \multicolumn{2}{c}{ Régimes } \\
\cline { 2 - 3 } Constituants $(\mathbf{g})$ & $\mathbf{R}_{\mathbf{1}}$ & $\mathbf{R}_{\mathbf{2}}$ \\
\hline Carica papaya & 16,68 & 14,28 \\
Lactuca sativa & 16,68 & 14,28 \\
Brassica oleracea & 16,68 & 14,28 \\
Cecropia peltata & 16,68 & 14,28 \\
Phaulopsis falcisepala & 16,68 & 14,28 \\
Laportea aestuans & 16,6 & 14,28 \\
leucena leucocephala & 00 & 14,32 \\
\hline Total (g) & $\mathbf{1 0 0}$ & $\mathbf{1 0 0}$ \\
\hline
\end{tabular}

Tableau 3: Composition biochimique des différents régimes alimentaires.

\begin{tabular}{lcccccc}
\hline $\begin{array}{l}\text { Régimes } \\
\text { alimentaires }\end{array}$ & $\begin{array}{c}\text { Humidité } \\
\mathbf{\%}\end{array}$ & $\begin{array}{c}\text { Protéines } \\
\mathbf{\%}\end{array}$ & $\begin{array}{c}\text { Matières } \\
\text { minérales } \\
\mathbf{\%}\end{array}$ & $\begin{array}{c}\text { Lipides } \\
\mathbf{( \% )}\end{array}$ & $\begin{array}{c}\text { Glucides } \\
\mathbf{\%}\end{array}$ & $\begin{array}{c}\text { Energie } \\
\text { métabolisable } \\
(\mathbf{K c a l} / \mathbf{1 0 0 g})\end{array}$ \\
\hline $\mathbf{R}_{\mathbf{1}}$ & $89,27^{\mathrm{a}}$ & $2,75^{\mathrm{e}}$ & $1,32^{\mathrm{c}}$ & $0,12^{\mathrm{b}}$ & $6,54^{\mathrm{b}}$ & $39,44^{\mathrm{b}}$ \\
$\mathbf{R}_{\mathbf{2}}$ & $76,5^{\mathrm{b}}$ & $4,94^{\mathrm{d}}$ & $2,98^{\mathrm{b}}$ & $0,17^{\mathrm{b}}$ & $4,33^{\mathrm{b}}$ & $41,08^{\mathrm{b}}$ \\
$\mathbf{R}_{\mathbf{3}}$ & $6,51^{\mathrm{c}}$ & $10,5^{\mathrm{c}}$ & $34,3^{\mathrm{a}}$ & $2,09^{\mathrm{a}}$ & $42,11^{\mathrm{a}}$ & $246,45^{\mathrm{a}}$ \\
$\mathbf{R}_{\mathbf{4}}$ & $6,42^{\mathrm{c}}$ & $14^{\mathrm{b}}$ & $36^{\mathrm{a}}$ & $2,19^{\mathrm{a}}$ & $40,58^{\mathrm{a}}$ & $241,71^{\mathrm{a}}$ \\
$\mathbf{R}_{\mathbf{5}}$ & $6,64^{\mathrm{c}}$ & $17,5^{\mathrm{a}}$ & $35,2^{\mathrm{a}}$ & $2,09^{\mathrm{a}}$ & $39,97^{\mathrm{a}}$ & $243,09^{\mathrm{a}}$ \\
\hline \multicolumn{2}{l}{}
\end{tabular}


N. A. SIKA PIBA et al. / Int. J. Biol. Chem. Sci. 8(5): 2296-2305, 2014

Tableau 4 : Ingestion alimentaire, taux moyen journalier de consommation et rendement écologique de croissance en fonction du régime et du taux de protéine alimentaire.

\begin{tabular}{|c|c|c|c|c|c|}
\hline \multirow[b]{2}{*}{ Variables } & \multicolumn{5}{|c|}{ Régimes alimentaires } \\
\hline & $\begin{array}{c}\mathbf{R}_{1} \\
(2,75 \% \text { de protéine })\end{array}$ & $\begin{array}{c}\mathbf{R}_{2} \\
(4,94 \% \text { de protéine })\end{array}$ & $\begin{array}{c}\mathrm{R}_{3} \\
(\mathbf{1 0 \%} \text { de protéine) }\end{array}$ & $\begin{array}{c}R_{4} \\
\text { (14\% de protéine) }\end{array}$ & $\begin{array}{c}R_{5} \\
\text { (17,5\%de protéine) }\end{array}$ \\
\hline Ingestion alimentaire $(\mathrm{g} / \mathrm{j} / \mathrm{escargot})$ & $1,89^{\mathrm{a}} \pm 0,75$ & $1,24^{\mathrm{a}} \pm 0,71$ & $0,78^{\mathrm{b}} \pm 0,44$ & $0,91^{\mathrm{b}} \pm 0,46$ & $1^{\mathrm{b}} \pm 0,44$ \\
\hline Taux moyen journalier de consommation (\%) & $6,45^{\mathrm{a}}$ & $5,79^{\mathrm{b}}$ & $2,26^{\mathrm{c}}$ & $2,03^{\mathrm{c}}$ & $2,19^{c}$ \\
\hline Rendement écologique de croissance (\%) & $16,37^{\mathrm{e}}$ & $22,5^{\mathrm{d}}$ & $48,33^{\mathrm{c}}$ & $50,61^{\mathrm{b}}$ & $61^{\mathrm{a}}$ \\
\hline
\end{tabular}

Les valeurs moyennes de la même ligne, indexées des mêmes lettres ne sont pas différentes.

Tableau 5: Poids des différentes parties du corps de A. fulica en fonction du régime et du taux de protéine alimentaire.

\begin{tabular}{|c|c|c|c|c|c|c|}
\hline Régimes alimentaires & Poids vifs (g) & $\begin{array}{l}\text { Quantité de coquilles } \\
\text { (g) }\end{array}$ & $\begin{array}{l}\text { Quantité de tissu mou } \\
\text { (g) }\end{array}$ & $\begin{array}{l}\text { Perte liée à la } \\
\text { Préparation (g) }\end{array}$ & $\begin{array}{l}\text { Quantité de } \\
\text { Viande (g) }\end{array}$ & $\begin{array}{l}\text { Quantité de } \\
\text { Viscère (g) }\end{array}$ \\
\hline $\mathrm{R}_{1}(2,75 \%$ protéine $)$ & $119,47^{\mathrm{e}} \pm 7,33$ & $24,98^{\mathrm{e}} \pm 2,32$ & $83,46^{\mathrm{e}} \pm 6,41$ & $11,03^{\mathrm{c}} \pm 2,18$ & $52,58^{\mathrm{e}} \pm 11,51$ & $30,87^{\mathrm{c}} \pm 11,16$ \\
\hline $\mathrm{R}_{2}$ (4,94\%protéine) & $133,67^{\mathrm{d}} \pm 6,16$ & $27,90^{\mathrm{d}} \pm 3,24$ & $93,68^{d} \pm 7,03$ & $12,08^{\mathrm{c}} \pm 2,18$ & $57,90^{\mathrm{d}} \pm 10,53$ & $35,78^{\mathrm{b}} \pm 5,1$ \\
\hline $\mathrm{R}_{3}(10,5 \%$ protéine $)$ & $148,97^{\mathrm{c}} \pm 9,74$ & $34,48^{\mathrm{c}} \pm 7,94$ & $102,23^{\mathrm{c}} \pm 12,82$ & $12,26^{\mathrm{c}} \pm 2,40$ & $62,88^{\mathrm{c}} \pm 9,29$ & $39,34^{b} \pm 9,16$ \\
\hline $\mathrm{R}_{4}(14 \%$ protéine $)$ & $178,53^{\mathrm{b}} \pm 8,73$ & $42,07^{b} \pm 5,08$ & $121,41^{\mathrm{b}} \pm 6,92$ & $15,02^{\mathrm{b}} \pm 3,70$ & $76,81^{\mathrm{b}} \pm 8,5$ & $44,61^{a} \pm 7,86$ \\
\hline $\mathrm{R}_{5}(17,5 \%$ protéine $)$ & $198,6^{\mathrm{a}} \pm 5,77$ & $49,03^{\mathrm{a}} \pm 4,31$ & $132,91^{\mathrm{a}} \pm 6,73$ & $16,67^{\mathrm{a}} \pm 3,78$ & $85,45^{\mathrm{a}} \pm 8,52$ & $47,46^{\mathrm{a}} \pm 6,18$ \\
\hline
\end{tabular}


N. A. SIKA PIBA et al. / Int. J. Biol. Chem. Sci. 8(5): 2296-2305, 2014

Tableau 6: Proportion du corps d'A. fulica en fonction du régime et du taux de protéine alimentaire.

\begin{tabular}{|c|c|c|c|c|c|c|}
\hline Régimes alimentaires & $\begin{array}{c}\% \\
\text { Poids total }\end{array}$ & $\begin{array}{c}\% \\
\text { coquilles }\end{array}$ & $\begin{array}{c}\% \\
\text { tissu mou }\end{array}$ & $\begin{array}{l}\text { \% Perte liée à } \\
\text { la préparation }\end{array}$ & $\begin{array}{c}\% \\
\text { viande }\end{array}$ & $\begin{array}{c}\% \\
\text { viscère }\end{array}$ \\
\hline $\mathrm{R}_{1}(2,75 \%$ protéine $)$ & 100 & $20,93^{b} \pm 1,80$ & $69,83^{\mathrm{a}} \pm 2,60$ & $9,23^{\mathrm{a}} \pm 1,74$ & $43,97^{\mathrm{a}} \pm 8,7$ & $25,87^{\mathrm{a}} \pm 9,08$ \\
\hline $\mathrm{R}_{2}$ (4,94\% protéine) & 100 & $20,93^{b} \pm 2,74$ & $70,03^{\mathrm{a}} \pm 3,13$ & $9,03^{\mathrm{a}} \pm 1,51$ & $43,27^{\mathrm{a}} \pm 7,23$ & $26,77^{\mathrm{a}} \pm 6,26$ \\
\hline $\mathrm{R}_{3}(10,5 \%$ protéine $)$ & 100 & $23,23^{a} \pm 5,53$ & $68,5^{\mathrm{ab}} \pm 6,08$ & $8,27^{\mathrm{b}} \pm 1,70$ & $42,2^{\mathrm{a}} \pm 5,64$ & $26,3^{a} \pm 5,36$ \\
\hline $\mathrm{R}_{4}(14 \%$ protéine $)$ & 100 & $23,57^{\mathrm{a}} \pm 2,40$ & $68,03^{\mathrm{b}} \pm 2,80$ & $8,4^{\mathrm{ab}} \pm 1,98$ & $43^{\mathrm{a}} \pm 4,09$ & $25,03^{\mathrm{a}} \pm 4,59$ \\
\hline $\mathrm{R}_{5}(17,5 \%$ protéine $)$ & 100 & $24,7^{\mathrm{a}} \pm 2,17$ & $66,9^{b} \pm 2,19$ & $8,4^{\mathrm{ab}} \pm 1,96$ & $43^{\mathrm{a}} \pm 3,81$ & $23,9^{\mathrm{a}} \pm 3,07$ \\
\hline
\end{tabular}

Tableau 7: Composition biochimique des masses viscérale et pédieuse d'A. fulica en fonction du régime et du taux de protéine alimentaire.

\begin{tabular}{|c|c|c|c|c|c|c|}
\hline Régimes & Teneur en protéine & Parties du corps & $\begin{array}{l}\text { Matière sèche } \\
\text { (g/100g) }\end{array}$ & $\begin{array}{l}\text { Protéine } \\
\text { (g/100g) }\end{array}$ & $\begin{array}{c}\text { Matières minérales } \\
(\mathrm{g} / \mathbf{1 0 0 g})\end{array}$ & Energie $(\mathrm{Cal} / \mathbf{1 0 0 g})$ \\
\hline \multirow[t]{2}{*}{ Naturel } & \multirow[t]{2}{*}{-} & Masse pédieuse & 18,54 & 67,9 & 7,56 & 4967 \\
\hline & & Masse viscérale & 23,65 & 61,49 & 8,86 & 4898 \\
\hline \multirow{2}{*}{$\mathbf{R}_{1}$} & \multirow{2}{*}{2,75} & Masse pédieuse & 21,02 & 58,96 & 4,02 & 5012 \\
\hline & & Masse viscérale & 24,94 & 52,88 & 6,18 & 4992 \\
\hline \multirow[t]{3}{*}{$\mathbf{R}_{2}$} & \multirow[t]{2}{*}{4,94} & Masse pédieuse & 29,52 & 60,53 & 3,95 & 4889 \\
\hline & & Masse viscérale & 21,94 & 56,12 & 5,94 & 4023 \\
\hline & \multirow[b]{2}{*}{10,5} & Masse pédieuse & 15,84 & 63,07 & 15,09 & 5101 \\
\hline $\mathbf{R}_{\mathbf{3}}$ & & Masse viscérale & 20,28 & 56,49 & 17,23 & 4865 \\
\hline \multirow[b]{2}{*}{$\mathbf{R}_{4}$} & \multirow[t]{2}{*}{14} & Masse pédieuse & 14,85 & 63,96 & 15,19 & 5224 \\
\hline & & Masse viscérale & 16,98 & 60,25 & 16,77 & 4969 \\
\hline \multirow[t]{2}{*}{$\mathbf{R}_{5}$} & \multirow[t]{2}{*}{17,5} & Masse pédieuse & 13,28 & 65,74 & 14,98 & 5406 \\
\hline & & Masse viscérale & 17,06 & 59,85 & 17,09 & 5002 \\
\hline
\end{tabular}




\section{DISCUSSION}

Les quantités moyennes des régimes constitués de fourrages verts consommées par escargot $\left(1,24 \mathrm{~g} / \mathrm{j} / \mathrm{escargot}\right.$ pour $\mathrm{R}_{1}$ et $1,89 \mathrm{~g} / \mathrm{j} /$ escargot pour $\mathrm{R}_{2}$ ) dans cette étude, sont largement supérieures à la quantité la plus élevée d'aliment concentré de farine consommée journellement par escargot (1 $\mathrm{g} / \mathrm{j} /$ escargot). Cela pourrait se justifier par le fait que les régimes végétaux étant pauvres en nutriments, l'escargot en consomme beaucoup plus pour chercher à satisfaire ses besoins alimentaires. Aussi, Achatina fulica est-il un escargot terrestre africain essentiellement végétarien (N'Da et al., 2004). Cependant, le rendement écologique de croissance des régimes végétaux de fourrages verts est négligeable devant celui des régimes concentrés. Cela a pour conséquence une production de viande plus importante chez les animaux soumis aux concentrés que chez ceux soumis aux fourrages verts. En effet, bien que les animaux soumis aux différents régimes alimentaires aient présenté sensiblement les mêmes pourcentages de coquille et de chair par rapport à leurs poids vifs, les quantités de tissu mou et de viande obtenues avec les régimes concentrés, sont nettement plus abondantes que celles obtenues avec les régimes constitués de fourrages verts. L'analyse des résultats montre que la quantité de viande produite par les escargots est liée au taux de protéine alimentaire. Ainsi, l'accroissement de la teneur en protéine des régimes alimentaires a pour conséquence une augmentation de la quantité de viande chez ces animaux. Ce résultat est en accord avec Otchoumou (2005) qui affirme que les croissances pondérale et coquillière sont étroitement liées à la teneur de certains nutriments comme les protéines. Pour une différence de $7,5 \%$ de protéine entre le régime concentré $\mathrm{R}_{3}(10 \%$ de protéine) et le régime concentré $\mathrm{R}_{4}$ (17,5\% de protéine), on obtient un gain de viande de 22,57 g. Les proportions de viande présentées par les escargots dans cette étude (42,2 à 43,97\%) sont largement supérieures à celles présentées par Otchoumou et al. (2010) chez la même espèce c'est-à-dire $26,61 \%$. Cette différence peut s'expliquer par une modification des conditions expérimentales surtout, au niveau des aliments et des substrats d'élevage utilisés au cours de ces expériences. Cela pourrait aussi se justifier par une différence entre les parties jugées de consommables au cours des deux études. Dans cette étude, la portion constituée du pied, de la tête, du bord distal du manteau portant le dernier centimètre du rectum et l'anus, du pneumostome, du bourrelet palléal, et de la partie distale du tractus génital y compris le manchon pénien, la partie antérieure du tractus digestif jusqu'à la première moitié du jabot a été jugée consommable (Aman, 2013).

Cette étude a montré aussi une variation de la composition biochimique des animaux en fonction du régime et du taux de protéine alimentaire. En effet, le taux de protéine, que ce soit dans la masse pédieuse que dans la masse viscérale est légèrement plus important chez les escargots soumis aux régimes concentrés plus riches en protéine que chez ceux soumis aux régimes constitués de fourrages verts. Cela suggère que la composition biochimique des escargots s'enrichie avec celle du régime alimentaire auquel ils sont soumis. Le taux de protéine dans la chair des escargots sauvages est légèrement plus important que ceux d'élevage soumis aux régimes végétaux comme aux régimes concentrés. Cela serait dû au fait que Achatina fulica dans la nature est une espèce herbivore polyphage. Elle est vorace et est capable de manger n'importe quoi, détritus, cadavres, feuilles, fruits, écorces, légumes; ce qui a une influence sur la composition biochimique de sa chair (Otchoumou et al., 2010).

\section{Conclusion}

Le rendement en viande ainsi que la composition biochimique de la chair de Achatina fulica sont fortement influencés 
par la teneur en protéines de son régime alimentaire. Les animaux soumis aux régimes constitués de fourrages verts ont présenté des quantités de chair consommables $\left(52,58 \pm 11,51 \mathrm{~g}\right.$ pour $\mathrm{R}_{1}$ et $57,90 \pm 10,53 \mathrm{~g}$ pour $\mathrm{R}_{2}$ ) plus faibles que celles de ceux soumis aux régimes concentrés de farine $\left[62,88 \pm 9,29 \mathrm{~g}\left(\mathrm{R}_{3}\right)\right.$; $76,81 \pm 8,50 \mathrm{~g}\left(\mathrm{R}_{4}\right)$ et $\left.85,45 \pm 8,52 \mathrm{~g}\left(\mathrm{R}_{5}\right)\right]$. Le régime $\mathrm{R}_{5}$ présentant la plus forte teneur en protéine $(17,5 \%)$, a permis d'obtenir la plus importante quantité de chair consommable $(85,45 \mathrm{~g})$. En revanche, la plus petite quantité de viande est fournie par les escargots soumis au régime végétal $\mathrm{R}_{1}$ sans Leucena leucocephala avec la plus faible teneur en protéine $(2,75 \%)$. Ainsi, la quantité de viande des escargots s'accroît avec la teneur en protéines du régime alimentaire auquel ils sont soumis.

\section{REFERENCES}

Ademolu KO, Idowu AB, Mafiang CF, Osinowo OA. 2004. Performance, proximate and mineral analysis of African giant land snail (A. marginata) fed with different nitrogen sources. African Journal of Biotechnology, 3(8): 412-417.

Aman J-B. 2013. Effet de la source et de la teneur en calcium du substrat d'élevage sur les performances biologiques d'Archachatina marginata (Swainson, 1821). Thèse de Doctorat unique en Biologie et Productions Animales, UFR des Sciences de la Nature, Université NANGUI ABROGOUA, Abidjan Côte d'Ivoire, $156 \mathrm{p}$.

Diomandé M, Kipré AV, Koussemon M, Kamenan A. 2008. Substitution de la farine de poisson par celle de l'escargot Achatina fulica dans l'alimentation des poules pondeuses en Côte d' Ivoire. Livestock Research for Rural Development, 20(1): http/: www.cipav.org.co/Irrd/Irrd20/1diom2 0002.htm.
Engmann FN, Afoakwah NA, Darko PO, Sefah W. 2013. Proximate and Mineral Composition of Snail (Achatina achatina) Meat; Any Nutritional Justification for Acclaimed Health Benefits? Journal of Basic and Applied Scientific Research, 3(4): 8-15.

Fagbuaro O, Oso JA, Edward JB, Ogunleye RF. 2006. Nutritional status of four species of giant land snails in Nigeria. Journal of Zhejiang University Science B, 7(9): 686-689.

Kouassi KD, Otchoumou A, Dosso $\mathrm{H}$. 2007. Effet de l'alimentation sur les performances biologiques chez l'escargot géant africain: Archachatina ventricosa (Gould, 1850) en élevage hors sol. Livestock Research for Rural Development, 19: 16-20.

N'Da K, Otchoumou A, Koffi KJC. 2004. Alimentation à base de produits du papayer et maturation ovocytaire chez Achatina fulica (Bowdich, 1720) en Côte d'Ivoire. Tropicultura, 22(4): 168-172.

Otchoumou A. 2005. Effet de la teneur en calcium d'aliments composés et de la photopériode sur les performances biologiques chez trois espèces d'escargots Achatinidae de Côte d'Ivoire élevées en bâtiment. Thèse de Doctorat d'Etat ES-Sciences Naturelles en Biologie et Ecologie Animales, Université d'AboboAdjamé, Abidjan Côte d'Ivoire, 171p.

Otchoumou A, Dupont-Nivet M, Ocho A, Atchibri L, Dosso H. 2010. Body proportions and chemical composition of wild and reared edible snails of Ivory Coast. Italian Journal of Food Science 22(1): 1120-1770.

Sami AS, Augustini C, Schwars FJ. 2004. Effects of feeding intensity and time on feed on performance, carcass characteristics and meat quality of Simmental bulls. Meat Sciences, 67: 195-201. 